\title{
The Disease Severity and Clinical Outcomes of the SARS-CoV-2 Variants of Concern
}

\author{
Lixin Lin ${ }^{1}$, Ying Liu ${ }^{2}$, Xiujuan Tang ${ }^{3}$ and Daihai He ${ }^{1 *}$ \\ ${ }^{1}$ Department of Applied Mathematics, The Hong Kong Polytechnic University, Kowloon, Hong Kong SAR, China, ${ }^{2}$ School of \\ International Business, Xiamen University Tan Kah Kee College, Zhangzhou, China, ${ }^{3}$ Shenzhen Center for Disease Control \\ and Prevention, Shenzhen, China
}

\section{OPEN ACCESS}

Edited by:

Pragya Dhruv Yadav,

National Institute of Virology (ICMR), India

Reviewed by:

Talha Bin Emran,

Begum Gulchemonara Trust

University, Bangladesh

Lin Wang,

University of Cambridge,

United Kingdom

*Correspondence:

Daihai He

daihai.he@polyu.edu.hk

Specialty section:

This article was submitted to Infectious Diseases-Surveillance,

Prevention and Treatment,

a section of the journal

Frontiers in Public Health

Received: 13 September 2021 Accepted: 08 November 2021

Published: 30 November 2021

Citation:

Lin L, Liu Y, Tang X and He D (2021)

The Disease Severity and Clinical

Outcomes of the SARS-CoV-2 Variants of Concern.

Front. Public Health 9:775224.

doi: 10.3389/fpubh.2021.775224
With the continuation of the pandemic, many severe acute respiratory syndrome coronavirus 2 (SARS-CoV-2) variants have appeared around the world. Owing to a possible risk of increasing the transmissibility of the virus, severity of the infected individuals, and the ability to escape the antibody produced by the vaccines, the four SARS-CoV-2 variants of Alpha (B.1.1.7), Beta (B.1.351), Gamma (P.1), and Delta (B.1.617.2) have attracted the most widespread attention. At present, there is a unified conclusion that these four variants have increased the transmissibility of SARS-CoV-2, but the severity of the disease caused by them has not yet been determined. Studies from June 1, 2020 to October 15, 2021 were considered, and a meta-analysis was carried out to process the data. Alpha, Beta, Gamma, and Delta variants are all more serious than the wild-type virus in terms of hospitalization, ICU admission, and mortality, and the Beta and Delta variants have a higher risk than the Alpha and Gamma variants. Notably, the random effects of Beta variant to the wild-type virus with respect to hospitalization rate, severe illness rate, and mortality rate are 2.16 (95\% Cl: 1.19-3.14), 2.23 (95\% Cl: 1.31-3.15), and 1.50 (95\% Cl: 1.26-1.74), respectively, and the random effects of Delta variant to the wild-type virus are 2.08 (95\% Cl: 1.77-2.39), 3.35 (95\% Cl: 2.5-4.2), and 2.33 (95\% Cl: 1.45-3.21), respectively. Although, the emergence of vaccines may reduce the threat posed by SARS-CoV-2 variants, these are still very important, especially the Beta and Delta variants.

Keywords: SARS-CoV-2, variants of concerns, disease severity, mortality, epidemic potential, COVID-19

\section{INTRODUCTION}

There are multiple severe acute respiratory syndrome coronavirus 2 (SARS-CoV-2) variants identified by viral genomic sequencing in different parts of the world. Based on the potential threats of these viral variants in terms of transmission, disease severity, immune escape, etc., they were classified into variants of concern (VOCs) and variants of interest (VOIs) by the World Health Organization (WHO). So far, four variants have been defined as VOCs-Alpha (B.1.1.7), Beta (B.1.351), Gamma (P.1), and Delta (B.1.617.2).

In late December 2020, the Alpha variant was reported in the United Kingdom (1), followed quickly by the detection of the Beta variant, which carried three mutations including K417N, E484K, and N501Y at important locations in the Spike protein receptor-binding domain (S-RBD) in South Africa (2). In early January 2021, the Gamma variant carrying three mutations consist of 
K417T, E484K, and N501Y in the S-RBD was reported in Brazil (3). In December 2020, the Delta variant carrying mutations called $452 \mathrm{R}$ and $478 \mathrm{~K}$ was first isolated in India (4).

Three key concerns of SARS-CoV-2 VOCs are viral transmissibility, disease severity, and the impacts on vaccine efficacy. For viral transmissibility, the reported studies have yielded good evidence that all VOCs are more transmissible than the wild-type virus (5-13). Risk of transmission, reported in 15 studies, was $45-71 \%$ higher for Alpha variant than the wild-type virus, while the basic reproduction number $R_{0 t}$ was $75-78 \%$ higher than the wild-type virus, and the reported effective reproduction number $R_{t}$ ranged from 1.1 to 2.8 (8). For Beta variant, the $R_{t}$ was 1.55 (95\% confidence interval [CI]: $1.43-1.69)$ and $\sim 50 \%$ more transmissible than the previously circulating variants $(11,12)$. Using dynamic modeling that integrates genomic and mortality data, Faria et al. (13) estimated that the transmissibility of the Gamma variant could be 1.4-2.2 times higher than that of the wild-type virus. A statistically significant increase in $R_{t}$ relative to wild-type virus of Delta variant at $97 \%$ (95\% CI: 76-117) (9). For impacts on vaccine effectiveness, the effects of the viral variants on the vaccine's protection of infection, symptomatic disease, and severe disease have been considered. The Alpha variant had less impact on the vaccine, and the vaccine was therefore still protective $(14,15)$. For Beta variant, the protection offered by the vaccine against symptomatic disease was reduced (16-18). The conclusion on the impact of Gamma variant on the vaccine was not yet clear. Delta variant likely reduced the protective effect of the vaccine with respect to infection and symptomatic disease (19).

Based on the newest report from WHO, the conclusions on disease severity were most uncertain among the reviews focusing on the phenotypic effects of SARS-CoV-2 VOCs. There were few reports on the disease severity of the variant viruses. Clinical outcomes were influenced by factors such as the use of health-care resource, demographic changes, and trends in social behavior (20). To date, we have found few reports of disease severity analysis based on clinical outcomes of the VOCs. By comparing four studies with datasets on the disease severity of infected persons, it was concluded that Alpha variant may not increase the risk of disease severity (21). A meta-analysis of these four studies indicated significantly increased hazard of mortality among patients with COVID-19 infected with Alpha variant relative to those infected with the wild-type virus (22). Alpha, Beta, and Gamma variants had a 1.7-, 3.6-, and 2.6-fold increased risk of hospitalization, and a 2.3-, 3.3-, and 2.2-fold increased risk of admission to the ICU, respectively (23). However, further

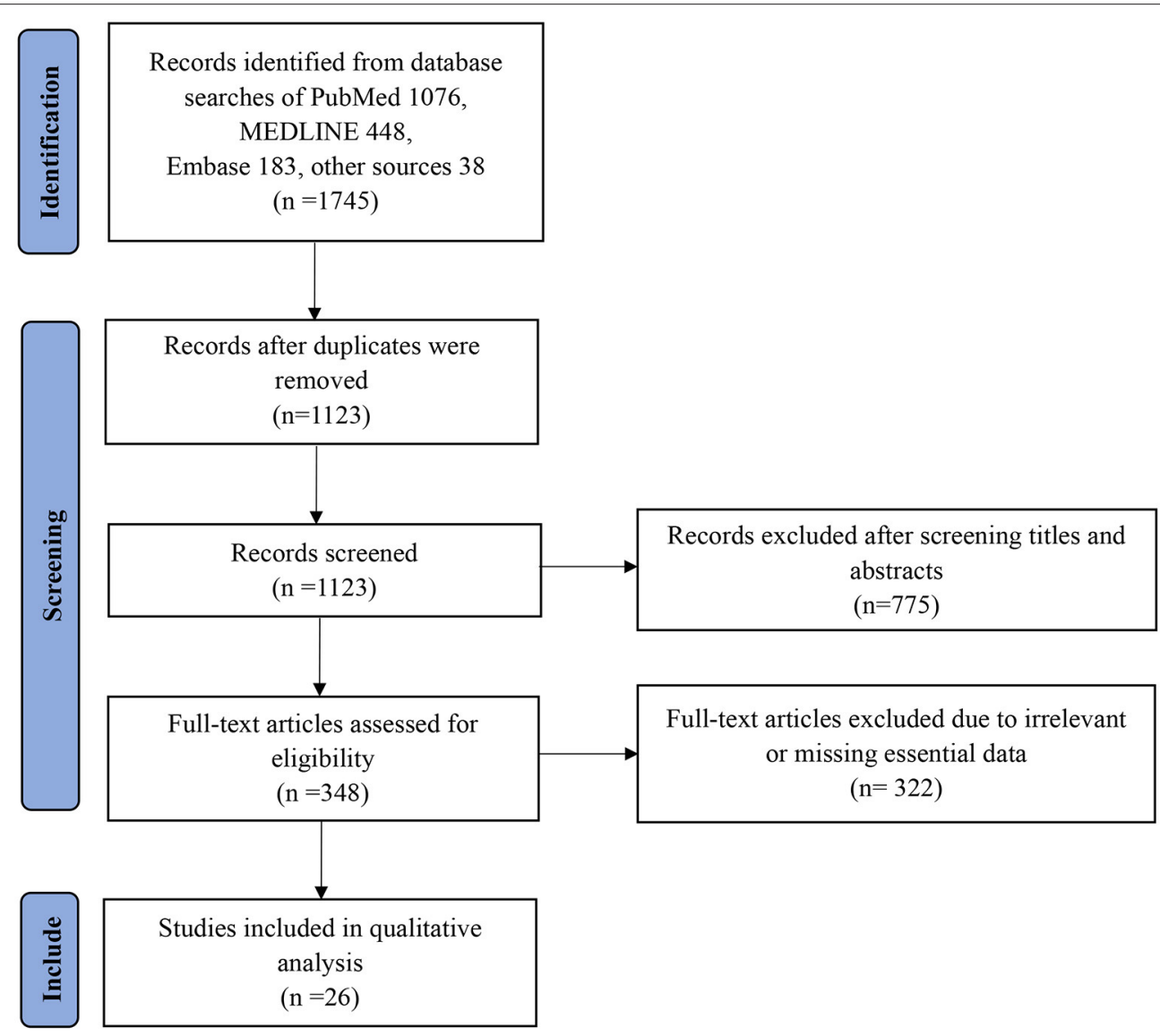

FIGURE 1 | Flow diagram of the search strategy and article selection process. 


\begin{tabular}{|c|c|c|c|c|c|c|c|c|c|c|}
\hline $\begin{array}{l}\text { The first author } \\
\text { (reference) }\end{array}$ & Variant type & $\begin{array}{l}\text { Patient } \\
\text { recruitment }\end{array}$ & Study dates & $\begin{array}{l}\text { Number of } \\
\text { participants }\end{array}$ & $\begin{array}{l}\text { Age of } \\
\text { participants, } \\
\text { years }\end{array}$ & Primary outcome & $\begin{array}{l}\text { Rate of Severe } \\
\text { disease }\end{array}$ & $\begin{array}{l}\text { Rate of } \\
\text { mortality }\end{array}$ & $\begin{array}{l}\text { Effect on severe } \\
\text { disease }\end{array}$ & $\begin{array}{l}\text { Effect on } \\
\text { mortality }\end{array}$ \\
\hline Frampton et al. (25) & Alpha & $\begin{array}{l}\text { Hospital } \\
\text { patients with } \\
\text { confirmed } \\
\text { COVID-19 }\end{array}$ & $\begin{array}{l}\text { November } 9 \\
\text { to December } \\
20,2020\end{array}$ & $\begin{array}{l}341(69 \%) \text { included } \\
\text { of } 496 \text { available } \\
\text { patients screened }\end{array}$ & $\begin{array}{l}\text { Median } 60 \\
\text { (IQR: 47-75) }\end{array}$ & $\begin{array}{l}\text { Clinical severity as } \\
\text { defined by } \mathrm{WHO} \\
\text { ordinal scale } \geq 6 \text {; } \\
\text { mortality at } 28 \text { days }\end{array}$ & 0.369 & 0.162 & $\begin{array}{l}\text { HR: } 1.02 \text { (95\% Cl: } \\
0.76-1.38)\end{array}$ & $\begin{array}{l}\text { HR: } 1.12(95 \% \mathrm{Cl} \\
0.71-1.78)\end{array}$ \\
\hline Challen et al. (26) & Alpha & $\begin{array}{l}\text { Public health } \\
\text { data from } \\
\text { community- } \\
\text { based testing } \\
\text { dataset }\end{array}$ & $\begin{array}{l}\text { Oct } 1,2020, \\
\text { to Jan } 28, \\
2021\end{array}$ & $\begin{array}{l}109,812(11.6 \%) \\
\text { included of } \\
941,518 \text { available } \\
\text { patients screened }\end{array}$ & $\begin{array}{l}\text { Mean } 46.3 \text { (SD } \\
11.0)\end{array}$ & Mortality at 28 days & NA & 0.003 & NA & $\begin{array}{l}\text { HR: } 1.64 \text { (95\% Cl: } \\
1.32-2.04)\end{array}$ \\
\hline Davies et al. (27) & Alpha & $\begin{array}{l}\text { Public health } \\
\text { data from } \\
\text { community- } \\
\text { based testing } \\
\text { dataset }\end{array}$ & $\begin{array}{l}\text { November } 1 \text {, } \\
2020 \text { to } \\
\text { January 23, } \\
2021\end{array}$ & $\begin{array}{l}1,146,534(51.1 \%) \\
\text { included of } \\
2,245,263 \text { available } \\
\text { patients screened }\end{array}$ & $\begin{array}{l}1-34(44.8 \%) ; \\
\text { 35-54 (35.2\%); } \\
\text { 55-69 (15.3\%); } \\
\text { 70-84 (3.8\%); } \\
\geq 85(0.8 \%)\end{array}$ & Mortality at 28 days & NA & 0.009 & NA & $\begin{array}{l}\text { HR: } 1.55 \text { (95\% Cl: } \\
1.39-1.72)\end{array}$ \\
\hline Grint et al. (28) & Alpha & $\begin{array}{l}\text { Public health } \\
\text { data from } \\
\text { community and } \\
\text { hospital-based } \\
\text { testing dataset }\end{array}$ & $\begin{array}{l}\text { November } \\
16,2020 \text { to } \\
\text { January 11, } \\
2021\end{array}$ & $\begin{array}{l}184,786(41.9 \%) \\
\text { included of } \\
441,161 \text { available } \\
\text { patients screened }\end{array}$ & $\begin{array}{l}\text { Median } 38.0 \\
\text { (IQR: } \\
\text { 24.0-52.0); } \\
\text { mean 38.2 (SD: } \\
\text { 18.1) }\end{array}$ & Mortality at 28 days & NA & 0.005 & NA & $\begin{array}{l}\text { HR: } 1.67 \text { (95\% Cl: } \\
1.34-2.09)\end{array}$ \\
\hline Patone et al. (29) & Alpha & $\begin{array}{l}\text { Public health } \\
\text { data from } \\
\text { community- } \\
\text { based testing } \\
\text { dataset }\end{array}$ & $\begin{array}{l}\text { November } 1 \text {, } \\
2020 \text { to } 26 \\
\text { January, } \\
2021\end{array}$ & $\begin{array}{l}80,494(40.6 \%) \\
\text { included of } \\
198,420 \text { available } \\
\text { patients screened }\end{array}$ & NA & $\begin{array}{l}\text { Clinical severity } \\
\text { reported by being } \\
\text { admitted to CCU: } \\
\text { Mortality at } 28 \text { days }\end{array}$ & NA & 0.008 & $\begin{array}{l}\text { HR: } 1.99 \text { (95\% Cl: } \\
1.59,2.49)\end{array}$ & $\begin{array}{l}\text { HR: } 1.59 \\
(1.25-2.03)\end{array}$ \\
\hline Loconsole et al. (30) & Alpha & $\begin{array}{l}\text { Public health } \\
\text { data from } \\
\text { community- } \\
\text { based testing } \\
\text { dataset }\end{array}$ & $\begin{array}{l}\text { December } \\
2020 \text { to } \\
\text { March } 2021\end{array}$ & $\begin{array}{l}621(20.2 \%) \\
\text { included of 3,075 } \\
\text { available patients } \\
\text { screened }\end{array}$ & $\begin{array}{l}0-4 \\
(3.38 \%) ; 5-16 \\
(12.08 \%) ; 17-35 \\
(23.19 \%) ; 36-65 \\
(43.8 \%) ;>65 \\
(17.55 \%)\end{array}$ & $\begin{array}{l}\text { Clinical severity } \\
\text { reported by being } \\
\text { admitted to Hospital, } \\
\text { as severe; } \\
\text { Mortality reported }\end{array}$ & $\begin{array}{l}\text { Hospital: } 5.6 \% \text {; } \\
\text { Severe: } 6.5 \%\end{array}$ & $0.6 \%$ & $\begin{array}{l}\text { HR } \\
\text { Hospital: 2; } \\
\text { Severe: } 1.27\end{array}$ & HR: 0.67 \\
\hline Funk et al. (31) & $\begin{array}{l}\text { Alpha and } \\
\text { Beta and } \\
\text { Gamma }\end{array}$ & $\begin{array}{l}\text { Public health } \\
\text { data from } \\
\text { community- } \\
\text { based testing } \\
\text { dataset }\end{array}$ & $\begin{array}{l}\text { October } \\
2020 \text { to } \\
\text { March } 2021\end{array}$ & $\begin{array}{l}23,343(0.7 \%) \\
\text { included of } \\
3,200,000 \text { available } \\
\text { patients screened }\end{array}$ & $\begin{array}{l}\text { Alpha: } \\
\text { Mean } 39 \text { (SD: } \\
\text { 21); } \\
\text { Beta: } \\
\text { Mean } 43 \text { (SD: } \\
\text { 22); } \\
\text { Gamma: } \\
\text { Mean } 46 \text { (SD: } \\
\text { 25); }\end{array}$ & $\begin{array}{l}\text { Clinical severity } \\
\text { reported by being } \\
\text { admitted to Hospital, } \\
\text { being admitted to ICU; } \\
\text { Mortality reported }\end{array}$ & $\begin{array}{l}\text { Hospital: 11\% } \\
\text { (Alpha); 19.3\% } \\
\text { (Beta); 20\% } \\
\text { (Gamma); ICU: } \\
\text { 1.4\% (Alpha); } \\
\text { 2.3\% (Beta); } 2.1 \% \\
\text { (Gamma) }\end{array}$ & $\begin{array}{l}0.02 \text { (Alpha); } \\
0.052 \text { (Beta); } \\
0.039 \\
\text { (Gamma) }\end{array}$ & $\begin{array}{l}\text { HR } \\
\text { Hospital (Alpha): } 1.7 \\
\text { (95\% Cl: 1.0-2.9), } \\
\text { (Beta): 3.6 (95\% Cl: } \\
\text { 2.1-6.2); (Gamma): } 2.6 \\
\text { (95\% Cl: } 1.4-4.8) ; \\
\text { ICU } \\
\text { (Alpha): } 2.3 \text { (95\% Cl: } \\
\text { 1.4-3.5); (Beta): 3.3 } \\
\text { (95\% Cl: 1.9-5.7); } \\
\text { (Gamma): } 2.2 \text { (95\% Cl: } \\
\text { 1.8-2.9) }\end{array}$ & $\begin{array}{l}\text { HR (Alpha): } 0.5 \\
\text { (95\% Cl: 0.3-0.9), } \\
\text { (Beta): 1.1 (95\% } \\
\text { Cl: 0.4-3.4), } \\
\text { (Gamma): } 0.6 \\
\text { (95\% Cl: 0.3-1.0) }\end{array}$ \\
\hline
\end{tabular}


TABLE 1 | Continued

\begin{tabular}{|c|c|c|c|c|c|c|c|c|c|c|}
\hline $\begin{array}{l}\text { The first author } \\
\text { (reference) }\end{array}$ & Variant type & $\begin{array}{l}\text { Patient } \\
\text { recruitment }\end{array}$ & Study dates & $\begin{array}{l}\text { Number of } \\
\text { participants }\end{array}$ & $\begin{array}{l}\text { Age of } \\
\text { participants, } \\
\text { years }\end{array}$ & Primary outcome & $\begin{array}{l}\text { Rate of Severe } \\
\text { disease }\end{array}$ & $\begin{array}{l}\text { Rate of } \\
\text { mortality }\end{array}$ & $\begin{array}{l}\text { Effect on severe } \\
\text { disease }\end{array}$ & $\begin{array}{l}\text { Effect on } \\
\text { mortality }\end{array}$ \\
\hline $\begin{array}{l}\text { Public Health } \\
\text { England (32) }\end{array}$ & $\begin{array}{l}\text { Alpha and } \\
\text { Delta }\end{array}$ & $\begin{array}{l}\text { Public health } \\
\text { data from } \\
\text { community- } \\
\text { based testing } \\
\text { dataset }\end{array}$ & $\begin{array}{l}\text { March, } 2021 \\
\text { to May, } 2021\end{array}$ & 38,805 & NA & $\begin{array}{l}\text { Clinical severity } \\
\text { reported by being } \\
\text { admitted to Hospital, } \\
\text { being admitted to } \\
\text { emergency care } \\
\text { attendance or } \\
\text { hospitalization }\end{array}$ & NA & NA & $\begin{array}{l}\text { HR } \\
\text { Hospital (Delta vs. } \\
\text { Alpha): 2.61, (95\% Cl: } \\
\text { 1.56-4.36); } \\
\text { care attendance or } \\
\text { hospitalization (Delta } \\
\text { vs. Alpha): } 1.67,(95 \% \\
\text { Cl: } 1.25-2.23)\end{array}$ & NA \\
\hline Bager et al. (33) & Alpha & $\begin{array}{l}\text { Public health } \\
\text { data from } \\
\text { community- } \\
\text { based testing } \\
\text { dataset }\end{array}$ & $\begin{array}{l}\text { January } 1 \text { to } \\
\text { March 24, } \\
2021\end{array}$ & $\begin{array}{l}10,544 \text { (20.7\%) } \\
\text { included of 50,958 } \\
\text { available patients } \\
\text { screened }\end{array}$ & $\begin{array}{l}0-29(44.4 \%) \\
30-59(44.3 \%) \\
\geq 60(11.3 \%)\end{array}$ & $\begin{array}{l}\text { Clinical severity } \\
\text { reported by being } \\
\text { admitted to hospital; } \\
\text { Mortality reported }\end{array}$ & 0.054 & NA & $\begin{array}{l}\text { HR } \\
\text { Hospital: } 1.42 \text { (95\% Cl: } \\
1.25-1.60)\end{array}$ & NA \\
\hline Cetin et al. (34) & Alpha & $\begin{array}{l}\text { Public health } \\
\text { data from } \\
\text { community- } \\
\text { based testing } \\
\text { dataset }\end{array}$ & $\begin{array}{l}\text { April } 2020 \text { to } \\
\text { March } 2021\end{array}$ & $\begin{array}{l}588(15.9 \%) \\
\text { included of 3,707 } \\
\text { available patients } \\
\text { screened }\end{array}$ & NA & $\begin{array}{l}\text { Clinical severity } \\
\text { reported by being } \\
\text { admitted to hospital, } \\
\text { being admitted to ICU; } \\
\text { Mortality reported }\end{array}$ & $\begin{array}{l}\text { Hospital: } 0.335 \text {, } \\
\text { ICU: } 0.075\end{array}$ & NA & $\begin{array}{l}\text { HR } \\
\text { Hospital: 2.62; ICU: } \\
1.923\end{array}$ & NA \\
\hline Fisman and Tuite (35) & $\begin{array}{l}\text { Alpha and } \\
\text { Beta and } \\
\text { Gamma and } \\
\text { Delta }\end{array}$ & $\begin{array}{l}\text { Public health } \\
\text { data from } \\
\text { community- } \\
\text { based testing } \\
\text { dataset }\end{array}$ & $\begin{array}{l}\text { February } 7 \\
\text { to June } 27 \\
2021\end{array}$ & $\begin{array}{l}\text { 168,909 (65.47\%) } \\
\text { included of } \\
257,997 \text { available } \\
\text { patients screened }\end{array}$ & NA & $\begin{array}{l}\text { Clinical severity } \\
\text { reported by being } \\
\text { admitted to hospital, } \\
\text { being admitted to ICU; } \\
\text { Mortality reported }\end{array}$ & $\begin{array}{l}\text { Hospital (Alpha } \\
\text { and Beta and } \\
\text { Gamma): 0.054, } \\
\text { ICU (Alpha and } \\
\text { Beta and Gamma): } \\
\text { 0.012; Hospital } \\
\text { (Delta): } 0.058, \text { ICU } \\
\text { (Delta): } 0.015\end{array}$ & $\begin{array}{l}0.009 \text { (Alpha } \\
\text { and Beta and } \\
\text { Gamma); } \\
0.007 \text { (Delta) }\end{array}$ & $\begin{array}{l}\text { HR } \\
\text { Hospital (Alpha and } \\
\text { Beta and Gamma): } \\
1.52 \text { (95\% Cl: } \\
\text { 1.42-1.63); (Delta): } \\
2.08 \text { (95\% Cl: } \\
\text { 1.78-2.4); } \\
\text { ICU } \\
\text { (Alpha and Beta and } \\
\text { Gamma): } 1.89 \text { (95\% Cl: } \\
\text { 1.67-2.17); (Delta): } \\
\text { 3.35 (95\% Cl: 2.6-4.3) }\end{array}$ & $\begin{array}{l}\text { HR (Alpha and } \\
\text { Beta and Gamma): } \\
\text { 1.51 (95\% Cl: } \\
\text { 1.3-1.78); (Delta): } \\
2.33 \text { (95\% Cl: } \\
\text { 1.54-3.31) }\end{array}$ \\
\hline Freitas et al. (36) & Gamma & $\begin{array}{l}\text { Public health } \\
\text { data from } \\
\text { community- } \\
\text { based testing } \\
\text { dataset }\end{array}$ & $\begin{array}{l}\text { April 1, } 2020 \\
\text { to May } 31, \\
2021 \text { and } \\
\text { January } 1 \text { to } \\
\text { January } 31, \\
2021\end{array}$ & $\begin{array}{l}6,142(47.4 \%) \\
\text { included of } 12,958 \\
\text { available patients } \\
\text { screened }\end{array}$ & NA & $\begin{array}{l}\text { Clinical severity } \\
\text { reported by being } \\
\text { admitted to hospital; } \\
\text { Mortality reported }\end{array}$ & Hospital: 0.860 & 0.597 & $\begin{array}{l}\text { HR } \\
\text { Hospital: } 0.914\end{array}$ & HR: 1.315 \\
\hline Grint et al. (37) & Alpha & $\begin{array}{l}\text { Public health } \\
\text { data from } \\
\text { community- } \\
\text { based testing } \\
\text { dataset }\end{array}$ & $\begin{array}{l}\text { November } \\
16,2020 \text { to } \\
\text { April } 21 \\
2021\end{array}$ & $\begin{array}{l}93,153(50.29 \%) \\
\text { included of } \\
185,234 \text { available } \\
\text { patients screened }\end{array}$ & NA & $\begin{array}{l}\text { Clinical severity } \\
\text { reported by being } \\
\text { admitted to hospital; } \\
\text { Mortality reported }\end{array}$ & 0.015 & 0.0027 & $\begin{array}{l}\text { HR: } 1.62 \text { (95\% Cl: } 1.48 \\
-1.78)\end{array}$ & $\begin{array}{l}\text { HR: } 1.73(95 \% \mathrm{Cl}: \\
1.41-2.13)\end{array}$ \\
\hline
\end{tabular}


TABLE 1 | Continued

\begin{tabular}{|c|c|c|c|c|c|c|c|c|c|c|}
\hline $\begin{array}{l}\text { The first author } \\
\text { (reference) }\end{array}$ & Variant type & $\begin{array}{l}\text { Patient } \\
\text { recruitment }\end{array}$ & Study dates & $\begin{array}{l}\text { Number of } \\
\text { participants }\end{array}$ & $\begin{array}{l}\text { Age of } \\
\text { participants, } \\
\text { years }\end{array}$ & Primary outcome & $\begin{array}{l}\text { Rate of Severe } \\
\text { disease }\end{array}$ & $\begin{array}{l}\text { Rate of } \\
\text { mortality }\end{array}$ & $\begin{array}{l}\text { Effect on severe } \\
\text { disease }\end{array}$ & $\begin{array}{l}\text { Effect on } \\
\text { mortality }\end{array}$ \\
\hline Giles et al. (38) & Alpha & $\begin{array}{l}\text { Hospitalized } \\
\text { patients with } \\
\text { confirmed } \\
\text { COVID-19 }\end{array}$ & NA & $\begin{array}{l}30(50 \%) \text { included } \\
\text { of } 60 \text { available } \\
\text { patients screened }\end{array}$ & NA & $\begin{array}{l}\text { Clinical severity as } \\
\text { defined by WHO } \\
\text { ordinal scale } \geq 6 \text {; } \\
\text { mortality at } 28 \text { day }\end{array}$ & 0.37 & 0.321 & HR: 1.37 & HR: 1.551 \\
\hline Hoang et al. (39) & $\begin{array}{l}\text { Alpha and } \\
\text { Beta and } \\
\text { Gamma }\end{array}$ & $\begin{array}{l}\text { Hospitalized } \\
\text { patients with } \\
\text { confirmed } \\
\text { COVID-19 }\end{array}$ & $\begin{array}{l}\text { February- } \\
\text { May 2020, } \\
\text { June- } \\
\text { December } \\
2020 \text {, } \\
\text { January } \\
\text {-September } \\
2021\end{array}$ & $\begin{array}{l}935(53.16 \%) \\
\text { included of 1,760 } \\
\text { available patients } \\
\text { screened }\end{array}$ & NA & $\begin{array}{l}\text { Clinical severity } \\
\text { reported by being } \\
\text { admitted to hospital, } \\
\text { being admitted to ICU; } \\
\text { Mortality reported }\end{array}$ & $\begin{array}{l}\text { Hospital (Alpha): } \\
\text { 0.249, (Beta): } \\
\text { 0.316, (Gamma): } \\
\text { 0.2; ICU (Alpha): } \\
\text { 0.071, (Beta): } \\
0.092 \text {, (Gamma): } \\
0.1\end{array}$ & $\begin{array}{l}\text { (Alpha): } \\
\text { 0.042, (Beta): } \\
\text { 0, (Gamma): } 0\end{array}$ & $\begin{array}{l}\text { HR } \\
\text { Hospital (Beta vs. } \\
\text { Alpha): } 1.27, \text { (Gamma } \\
\text { vs. Beta): } 0.633, \\
\text { (Gamma vs. Alpha): } \\
0.833 ; \text { ICU (Beta vs. } \\
\text { Alpha): } 1.314, \text { (Gamma } \\
\text { vs. Beta): } 1.087, \\
\text { (Gamma vs. Alpha): } \\
1.314\end{array}$ & NA \\
\hline Kim et al. (40) & Alpha & $\begin{array}{l}\text { Public health } \\
\text { data from } \\
\text { community- } \\
\text { based testing } \\
\text { dataset }\end{array}$ & $\begin{array}{l}\text { September } \\
20 \text { to } \\
\text { December } \\
15,2020\end{array}$ & $\begin{array}{l}1,769(50 \%) \\
\text { included of } 3,538 \\
\text { available patients } \\
\text { screened }\end{array}$ & NA & $\begin{array}{l}\text { Clinical severity } \\
\text { reported by being } \\
\text { admitted to hospital; } \\
\text { Mortality reported }\end{array}$ & Hospital: 0.009 & 0.0089 & HR: 0.6 & HR: 1.22 \\
\hline Meyer et al. (41) & Alpha & $\begin{array}{l}\text { Public health } \\
\text { data from } \\
\text { community- } \\
\text { based testing } \\
\text { dataset }\end{array}$ & $\begin{array}{l}\text { January } 12 \\
\text { to June } 3 \text {, } \\
2021\end{array}$ & $\begin{array}{l}59(1.66 \%) \\
\text { included of 3,544 } \\
\text { available patients } \\
\text { screened }\end{array}$ & $\begin{array}{l}\text { Minimum } 0.0 \\
\text { years, } \\
\text { maximum } 17.8 \\
\text { years }\end{array}$ & $\begin{array}{l}\text { Clinical severity } \\
\text { reported by being } \\
\text { admitted to hospital, } \\
\text { being admitted to ICU }\end{array}$ & $\begin{array}{l}\text { Hospital: 0.153; } \\
\text { ICU: } 0.017\end{array}$ & NA & $\begin{array}{l}\text { HR } \\
\text { Hospital: 1.89; } \\
\text { ICU: } \\
\text { NA }\end{array}$ & NA \\
\hline Ong et al. (42) & $\begin{array}{l}\text { Alpha, Beta, } \\
\text { and Delta }\end{array}$ & $\begin{array}{l}\text { The Ministry of } \\
\text { Health }\end{array}$ & $\begin{array}{l}\text { January } 1 \text { to } \\
\text { May 22, } \\
2021\end{array}$ & $\begin{array}{l}829(85 \%) \text { included } \\
\text { of } 976 \text { available } \\
\text { patients screened }\end{array}$ & NA & $\begin{array}{l}\text { Clinical severity } \\
\text { reported by being } \\
\text { admitted to ICU: } \\
\text { Mortality reported }\end{array}$ & NA & NA & $\begin{array}{l}\text { HR (Delta VS } \\
\text { wild-type); ICU: } 1.88 \\
\text { (95\% Cl: 0.95-3.76); } \\
\text { others No significant } \\
\text { difference }\end{array}$ & $\begin{array}{l}\text { HR (Delta vs. } \\
\text { wild-type): } 1.88 \\
\text { (95\% Cl: } \\
0.95-3.76) ; \text { others } \\
\text { No significant } \\
\text { difference }\end{array}$ \\
\hline $\begin{array}{l}\text { Martínez-García et al. } \\
\text { (43) }\end{array}$ & Alpha & $\begin{array}{l}\text { Hospital } \\
\text { patients with } \\
\text { confirmed } \\
\text { COVID-19 }\end{array}$ & $\begin{array}{l}\text { January } 2 \text { to } \\
\text { April 30, } \\
2021\end{array}$ & $\begin{array}{l}426(27.4 \%) \\
\text { included of 1,555 } \\
\text { available patients } \\
\text { screened }\end{array}$ & NA & $\begin{array}{l}\text { Clinical severity } \\
\text { reported by being } \\
\text { admitted to ICU; } \\
\text { Mortality reported }\end{array}$ & $19.5 \%$ & $13.9 \%$ & $\begin{array}{l}\text { HR } \\
\text { ICU: } 2.11(95 \% \mathrm{Cl}: \\
1.55-2.87)\end{array}$ & $\begin{array}{l}\text { HR: } 0.87 \text { (95\% Cl: } \\
0.62-1.23)\end{array}$ \\
\hline
\end{tabular}


TABLE 1 | Continued

\begin{tabular}{|c|c|c|c|c|c|c|c|c|c|c|}
\hline $\begin{array}{l}\text { The first author } \\
\text { (reference) }\end{array}$ & Variant type & $\begin{array}{l}\text { Patient } \\
\text { recruitment }\end{array}$ & Study dates & $\begin{array}{l}\text { Number of } \\
\text { participants }\end{array}$ & $\begin{array}{l}\text { Age of } \\
\text { participants, } \\
\text { years }\end{array}$ & Primary outcome & $\begin{array}{l}\text { Rate of Severe } \\
\text { disease }\end{array}$ & $\begin{array}{l}\text { Rate of } \\
\text { mortality }\end{array}$ & $\begin{array}{l}\text { Effect on severe } \\
\text { disease }\end{array}$ & $\begin{array}{l}\text { Effect on } \\
\text { mortality }\end{array}$ \\
\hline Yilmaz et al. (44) & Alpha & $\begin{array}{l}\text { Public health } \\
\text { data from } \\
\text { community- } \\
\text { based testing } \\
\text { Data set }\end{array}$ & $\begin{array}{l}\text { February } 2 \\
\text { to February } \\
9,2021\end{array}$ & $\begin{array}{l}339(26.1 \%) \\
\text { included of } 1,300 \\
\text { available patients } \\
\text { screened }\end{array}$ & NA & $\begin{array}{l}\text { Clinical severity } \\
\text { reported by being } \\
\text { admitted to Hospital; } \\
\text { in intensive care }\end{array}$ & $\begin{array}{l}\text { Hospital: } 3.2 \% \text {, } \\
\text { intensive care: } \\
0.58 \%\end{array}$ & NA & $\begin{array}{l}\text { HR } \\
\text { Hospital: } 47.76 \% \text {; } \\
\text { intensive care: } 77.78 \%\end{array}$ & NA \\
\hline Twohig et al. (45) & Alpha, Delta & $\begin{array}{l}\text { The Ministry of } \\
\text { Health }\end{array}$ & $\begin{array}{l}\text { January } 1 \text { to } \\
\text { May 22, } \\
2021\end{array}$ & $\begin{array}{l}829(85 \%) \text { included } \\
\text { of } 976 \text { available } \\
\text { patients screened }\end{array}$ & NA & $\begin{array}{l}\text { Clinical severity } \\
\text { reported by being } \\
\text { admitted to ICU; } \\
\text { Mortality reported }\end{array}$ & $\begin{array}{l}\text { Hospital: } 2.3 \% \text {, } \\
\text { emergency care: } \\
3.4 \%\end{array}$ & NA & $\begin{array}{l}\text { HR } \\
\text { Hospital (Delta vs. } \\
\text { Alpha): } 2.26 \text { ( } 95 \% \text { Cl: } \\
\text { 1.32-3.89); } \\
\text { emergency care: } 1.7\end{array}$ & NA \\
\hline Veneti et al. (46) & Alpha, Beta & $\begin{array}{l}\text { Norwegian } \\
\text { Surveillance } \\
\text { System for } \\
\text { Communicable } \\
\text { Diseases }\end{array}$ & $\begin{array}{l}\text { December } \\
28 \text { to May 2, } \\
2021\end{array}$ & $\begin{array}{l}23,717 \text { ( } 83.8 \%) \\
\text { included of } 28,301 \\
\text { available patients } \\
\text { screened }\end{array}$ & NA & $\begin{array}{l}\text { Clinical severity } \\
\text { reported by being } \\
\text { admitted to hospital, } \\
\text { being admitted to ICU }\end{array}$ & $\begin{array}{l}\text { Hospital (Alpha): } \\
\text { 3.8\%, (Beta): } \\
\text { 4.2\%, ICU (Alpha): } \\
\text { 0.8\%, (Beta): } 0.9 \%\end{array}$ & NA & $\begin{array}{l}\text { HR } \\
\text { Hospital (Alpha vs. } \\
\text { wild-type): } 1.9 \text { (95\% Cl: } \\
\text { 1.6-2.3), (Beta vs. } \\
\text { wild-type): } 2.4 \text { (95\% Cl: } \\
\text { 1.7-3.3); } \\
\text { ICU } \\
\text { (Alpha vs. wild-type): } \\
\text { 1.8 (95\% Cl: 1.2-2.8), } \\
\text { (Beta vs. wild-type): } 2.7 \\
\text { (95\% Cl: } 1.2-6.5)\end{array}$ & NA \\
\hline Patone et al. (47) & Alpha & $\begin{array}{l}\text { Public health } \\
\text { data from } \\
\text { community- } \\
\text { based testing } \\
\text { Data set }\end{array}$ & $\begin{array}{l}\text { November } 1 \text {, } \\
2020 \text { to } \\
\text { January } 27 \\
2021\end{array}$ & $\begin{array}{l}\text { 117,926 (59.4\%) } \\
\text { included of } \\
198,420 \text { available } \\
\text { patients screened }\end{array}$ & NA & $\begin{array}{l}\text { Clinical severity } \\
\text { reported by being } \\
\text { admitted to CCU; } \\
\text { mortality at } 28 \text { day }\end{array}$ & CCU: $0.4 \%$ & $0.4 \%$ & $\begin{array}{l}\text { HR } \\
\text { CCU: } 2.15 \text { (95\% Cl: } \\
1.75-2.65)\end{array}$ & $\begin{array}{l}\text { HR } 1.65 \text { (95\% Cl: } \\
1.36-2.01)\end{array}$ \\
\hline Nyberg et al. (48) & Alpha & $\begin{array}{l}\text { Public health } \\
\text { data from } \\
\text { community- } \\
\text { based } \\
\text { testing }\end{array}$ & $\begin{array}{l}\text { November } 1 \text {, } \\
2020 \text { to } \\
\text { January } 27 \text {, } \\
2021\end{array}$ & $\begin{array}{l}592,409 \text { (70.59\%) } \\
\text { included of } \\
839,278 \text { available } \\
\text { patients screened }\end{array}$ & NA & $\begin{array}{l}\text { Clinical severity } \\
\text { reported by being } \\
\text { admitted to Hospital; } \\
\text { mortality at } 28 \text { day }\end{array}$ & Hospital: $4.7 \%$ & $0.44 \%$ & $\begin{array}{l}\text { HR } \\
\text { Hospital: } 1.52 \text { (95\% Cl: } \\
1.47-1.57)\end{array}$ & $\begin{array}{l}\text { HR: } 1.59(95 \% \mathrm{Cl}: \\
1.44-1.74)\end{array}$ \\
\hline Stirrup et al. (49) & Alpha & $\begin{array}{l}\text { Hospital } \\
\text { patients with } \\
\text { confirmed } \\
\text { COVID-19 }\end{array}$ & $\begin{array}{l}\text { November } \\
16,2020 \text { to } \\
\text { January } 10 \\
2021\end{array}$ & $\begin{array}{l}1,107(47.29 \%) \\
\text { included of } 2,341 \\
\text { available patients } \\
\text { screened }\end{array}$ & NA & $\begin{array}{l}\text { Clinical severity } \\
\text { reported by being } \\
\text { admitted to ITU; } \\
\text { mortality at } 28 \text { day }\end{array}$ & ITU: $20.35 \%$ & $19.62 \%$ & $\begin{array}{l}\text { HR } \\
\text { ITU: } 1.01 \text { (95\% Cl: } \\
0.75-1.37)\end{array}$ & $\begin{array}{l}\text { HR: } 1.01 \text { (95\% Cl: } \\
0.79-1.28)\end{array}$ \\
\hline Whittaker et al. (50) & Alpha & $\begin{array}{l}\text { Public health } \\
\text { data from } \\
\text { community- } \\
\text { based testing } \\
\text { Data set }\end{array}$ & $\begin{array}{l}\text { December } \\
21,2020 \text { to } \\
\text { April 25, } \\
2021\end{array}$ & $\begin{array}{l}946(81 \%) \text { included } \\
\text { of } 1,186 \text { available } \\
\text { patients screened }\end{array}$ & NA & $\begin{array}{l}\text { Clinical severity } \\
\text { reported by being } \\
\text { admitted to ICU; Died } \\
\text { in hospital }\end{array}$ & ICU: 18\% & $6 \%$ & $\begin{array}{l}\text { HR } \\
\text { ICU: } 1.125\end{array}$ & HR: 1 \\
\hline
\end{tabular}


confirmation in larger studies of Alpha variant as well as other viral variants are needed.

\section{METHODS}

This study is a systematic review of current evidence conducted in June 2021 to determine the effects of SARS-CoV-2 VOCs on disease severity and clinical outcomes. The study was guided by the Preferred Reporting Items for Systematic Review and MetaAnalysis Protocols (PRISMA-P) to ensure reliability and validity of the reported results (24).

\section{Sources of Data}

A systematic search was conducted by using search terms in online databases such as PubMed, Medline, and Embase to retrieve all relevant English papers and reports published between June 1, 2020 and October 15, 2021. The search strategy adopted a combination of the following search terms: (B.1.1.7) OR (B.1.351) OR (P.1) OR (B.1.617.2) OR (SARS-CoV-2 Variants of Concern) OR (SARS-CoV-2 VOCs). Related references were also searched in Google Scholar.

\section{Selection of Research}

In all, 1,745 papers were extracted, and the full-text of the most relevant papers based on eligibility criteria were reviewed. Original and peer-reviewed papers in English that met the eligibility criteria in the final report were included. A flow chart of the search strategy and study selection process using PRISMA guidelines is presented in Figure 1. In addition, the following exclusion criteria were used:

- Non-human studies, including animal experiments, in vitro observations, and papers that do not refer to the keywords in this review.

- Papers that do not contain data on at least two kinds of viruses.

- The full paper is not available.

- Any duplicate and suspicious results in the database.

\section{Extraction of Data}

The first author's name, variant type, patient recruitment type, study dates, number of participants, age, rate of severe disease, and mortality were recorded in an information sheet. We rechecked the collected data to avoid duplication or overlap. Then, we extracted the relevant data (Table 1).

\section{Assessment of Quality}

This study adhered to the PRISMA guidelines to ensure the quality and accuracy of selected publications and outcomes.

\section{RESULTS}

We identified a total of 1,745 (1,076, PubMed; 448, MEDLINE; 183, Embase; and 38 from other sources) relevant articles, and 1,123 studies were left after removing the duplicates. After excluding 775 articles by title and abstract screening, 348 articles met the conditions for full-text screening. Based on the above exclusion criteria, a further 322 articles were excluded. Ultimately, 26 studies that met the inclusion criteria were selected in this review for further analysis. Each study's main findings are summarized in Table 1 (25-50). Among the 26 studies, most were related to variant Alpha, followed by Beta, Gamma, and Delta.

Through meta-analysis, the data provided by the retained studies were integrated; the values of total random effects were retained; and the risk of hospitalization, ICU admission, and mortality of patients infected with VOCs compared with wildtype virus were obtained to analyze the disease severity of the VOCs. The main process of meta-analysis of variants Alpha, Beta, Gamma, and Delta are, respectively, shown in Figures 2-5, and the main results of the meta-analysis are summarized in Table 2.

In the case of Alpha variant compared with the wild-type virus, most studies concluded that the risk of hospitalization, ICU admission, and mortality were increased. Only Frampton et al. (25) and Stirrup et al. (49) reported that the risk of ICU admission were equivalent. In addition, the differences in the risk of mortality mainly came from Funk et al. (31) and MartínezGarcía et al. (43) wherein they concluded that the mortality rate was reduced, and Stirrup et al. (49) concluded that the mortality rate was equivalent. For the Beta variant compared to wildtype virus, Funk et al. (31), Fisman and Tuite, (35), and Veneti et al. (46) found that it increased the risk of hospitalization and ICU admission. In addition, Funk et al. (31) and Fisman and Tuite, (35) reported that Beta variant also increased the risk of mortality. It is worth mentioning that Beta variant had the highest risk of hospitalization at 2.16 (95\% CI: 1.19-3.14). The only study involved in the meta-analysis regarding the Delta variant was from Fisman and Tuite, (35). The risk of ICU admission and mortality were 3.35 (95\% CI: 2.5-4.2) and 2.33 (95\% CI: 1.45-3.21) respectively, and it was slightly lower than that of Beta variant in the risk of hospitalization at $2.08(95 \%$ CI: 1.77-2.39)(35).

The results showed that in the risk of hospitalization, ICU admission, and mortality, all the SARS-CoV-2 VOCs had different degrees of increase compared with wild-type virus; Delta variant had the highest risk of ICU admission and mortality, and Beta variant had the highest risk of hospitalization.

\section{DISCUSSION}

Since the rapid spread of the SARS-COV-2 pandemic, many variant viruses including Alpha, Beta, Gamma, and Delta have emerged. However, the conclusions regarding disease severity of these variant viruses are not consistent. Accordingly, we searched for studies in the relevant field and recorded their clinical data. A meta-analysis was used to combine the information of different studies. Finally, we found that all VOCs increase the risk of hospitalization, ICU admission, and death compared with the wild-type virus, and variant Delta and Beta carried a much higher risk than other variants.

By comparing the results from different studies, we found that most of the conclusions stated that Alpha variant had a higher risk of disease severity than the wild-type virus, but Frampton et al. (25), Funk et al. (31), Martínez-García et al. (43), and Stirrup et al. (49) have expressed different opinions. However, the sample size of Frampton et al. (25) was very small, which is why their 


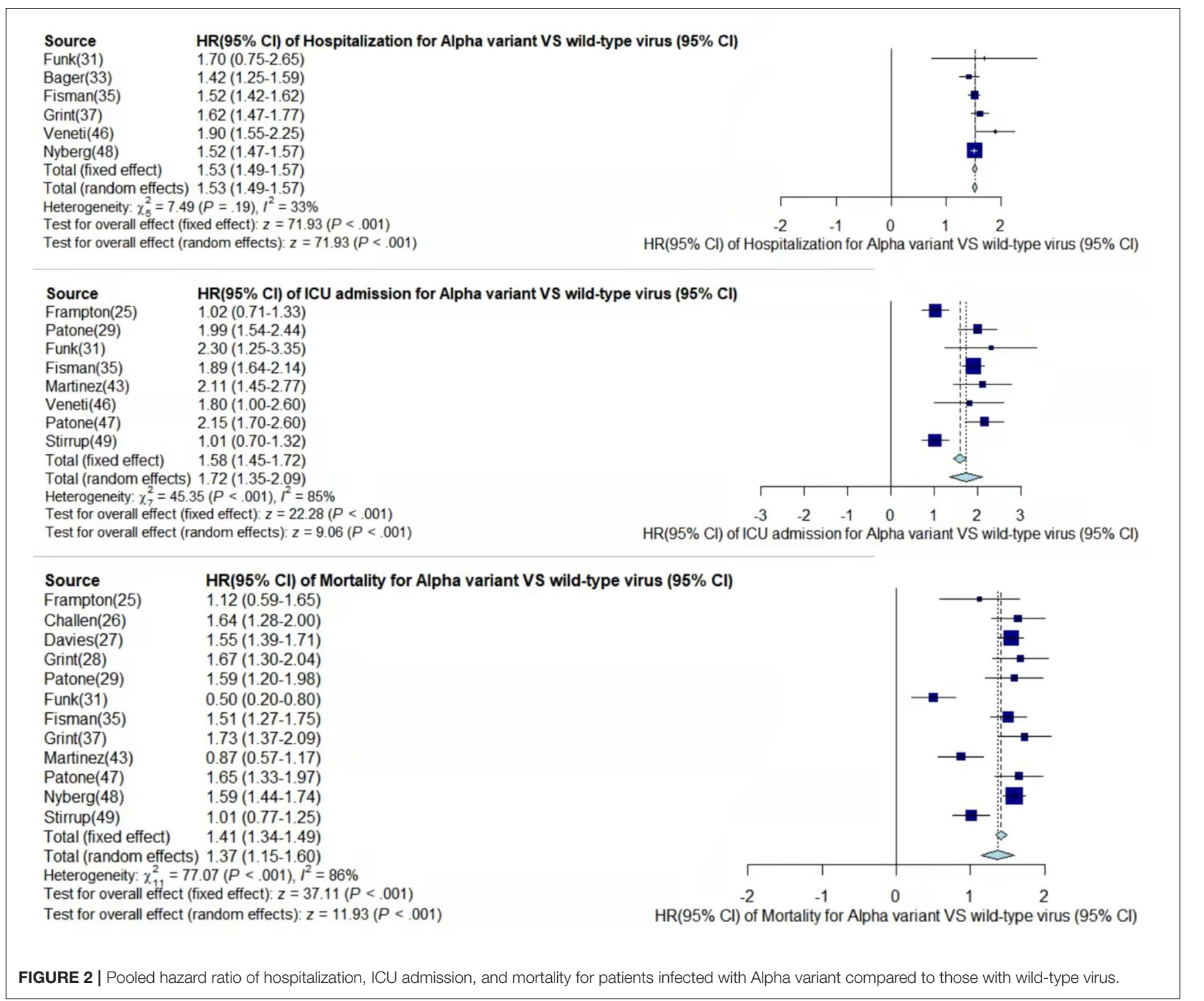

results were likely not very convincing. Although Frampton et al. (25) used whole genome sequencing to identify Alpha variant, while other reports used PCR detection of S-gene target failure (SGTF) as an alternative detection method, it did not make much of a difference to the results. Funk et al. (31) found that Alpha variant showed significantly higher risk of hospitalization rate and ICU admission, but lower risk of mortality than the wildtype. The clinical drugs for the Alpha variant in EU/EEA were more effective or some of the reported cases may have been vaccinated. The previously reported increased binding affinity between the spike receptor-binding domain and the angiotensinconverting enzyme 2 (ACE2) receptor in the Alpha SARS-CoV2 strain may have led to further down-regulation of ACE2 if an individual got infected by this new variant compared with other variants. ACE2 was suggested to have a protective effect on lung injury in patients with COVID-19 (51, 52). Patients were aged $\sim 70$ years, and there was a large gap with the age of other studies' patients, which led to certain limitations regarding the conclusion of mortality risk (43). Stirrup et al. (49) concluded that female rather than male patients infected with the Alpha variant would have a higher risk of severe disease. In summary, it can be argued that Alpha variant was more threatening than the wild-type virus and can cause higher risk of more severe disease. The explanation for the conclusion from Funk et al. (31) that variants Beta and Gamma may also have a lower risk of mortality can be consistent with the explanation for Alpha variant. In addition, Hoang et al. (39) speculated about the risk of hospitalization and ICU admission by directly comparing variants Beta, Gamma, and Alpha. The risk of Beta variant was significantly higher than that of Alpha variant, while the risk of Alpha variant was similar to that of Gamma variant, which was consistent with our conclusion.

Although the sample size on the Delta variant was limited in conducting meta-analysis, those studies that directly compared the disease severity of variants Delta and Alpha supported our conclusions from the side. Using stratified Cox proportional 

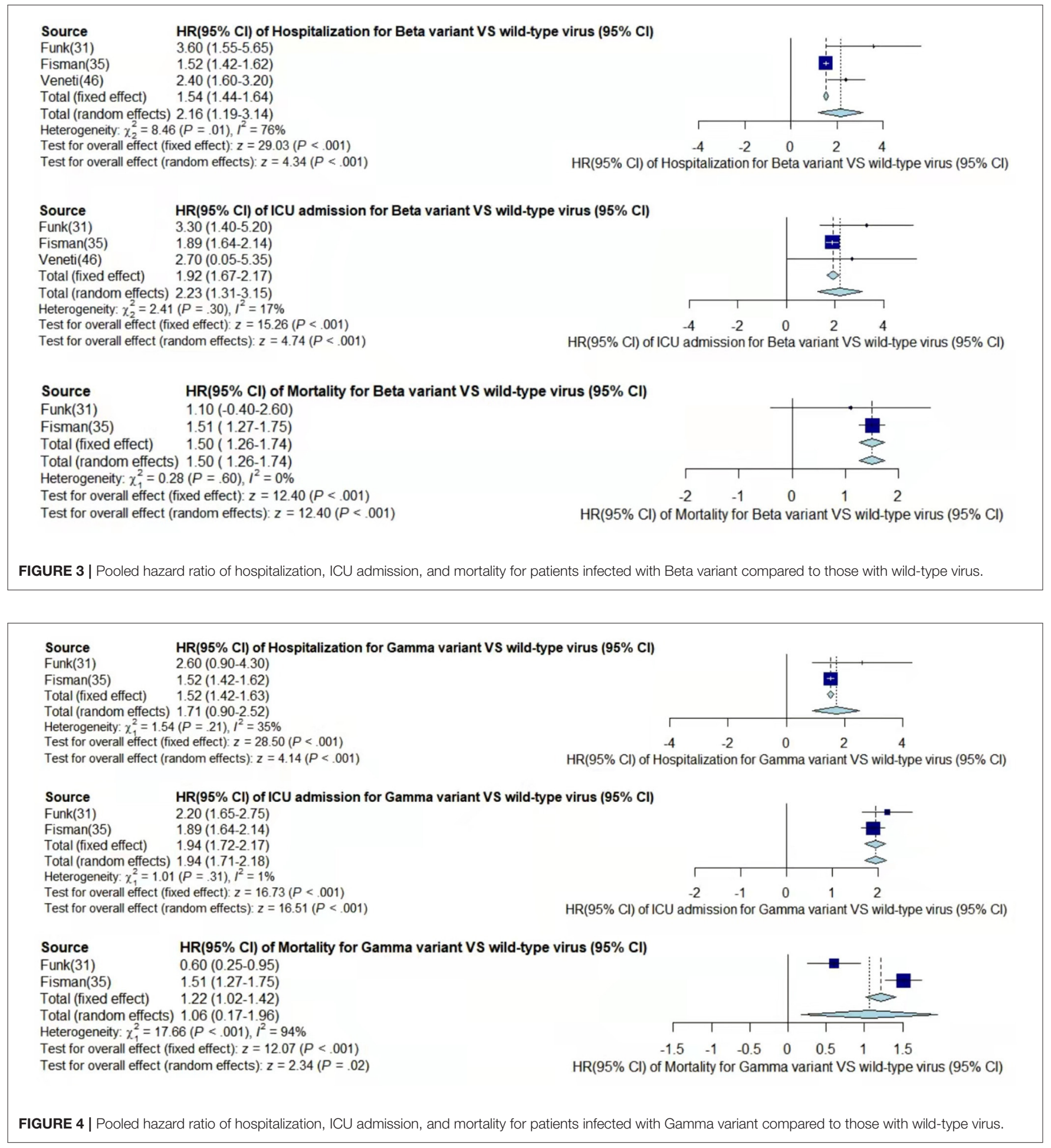

hazard regression, there was a significantly increasing risk of hospitalization and emergency care attendance for Delta variant cases compared with Alpha variant cases after adjustment for confounders, which were 2.16 (95\% CI: 1.56-4.36) and 1.67 (95\% CI: 1.25-2.23), respectively (32). Ong et al. (42) calculated that the risk of ICU admission and mortality were 1.88
(95\% CI: 0.95-3.76) and 1.88 (95\% CI: 0.95-3.76), respectively. Among similar studies, the main debate involved the study from Frampton et al. (25), but as mentioned earlier, their work was limited by a much smaller sample size, which is why their conclusions were not very persuasive (21). The result of Kow et al. (22) in the risk of mortality for Alpha variant compared 
Source $\quad H R(95 \% \mathrm{Cl})$ of Hospitalization for Delta variant VS wild-type virus $(95 \% \mathrm{Cl})$ Fisman(35) 2.08 (1.77-2.39)

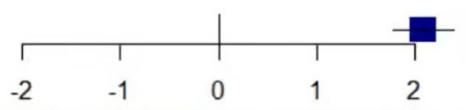

$\mathrm{HR}(95 \% \mathrm{Cl})$ of Hospitalization for Delta variant VS wild-type virus $(95 \% \mathrm{Cl})$

Source $\quad H R(95 \% \mathrm{CI})$ of ICU admission for Delta variant VS wild-type virus $(95 \% \mathrm{CI})$ Fisman(35) 3.35 (2.5-4.2)

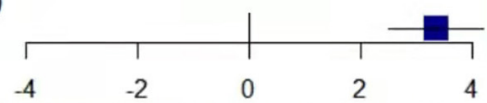

$\mathrm{HR}(95 \% \mathrm{Cl})$ of ICU admission for Delta variant VS wild-type virus (95\% Cl)

Source $\quad H R(95 \% \mathrm{Cl})$ of Mortality for Delta variant VS wild-type virus $(95 \% \mathrm{CI})$ Fisman(35) $2.33(1.45-3.21)$

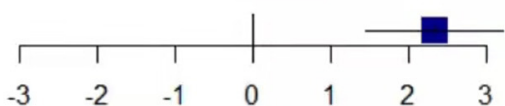

$\mathrm{HR}(95 \% \mathrm{Cl})$ of Mortality for Delta variant VS wild-type virus $(95 \% \mathrm{Cl})$

FIGURE 5 | Pooled hazard ratio of hospitalization, ICU admission, and mortality for patients infected with Delta variant compared to those with wild-type virus.

TABLE 2 | Hazard ratios (95\% Cl) of disease severity of the SARS-CoV-2 VOCs compared with wild-type virus.

\begin{tabular}{|c|c|c|c|c|}
\hline & Alpha & Beta & Gamma & Delta \\
\hline Risk of hospitalization & 1.53 (95\% Cl: 1.49-1.57) & 2.16 (95\% Cl: 1.19-3.14) & 1.71 (95\% Cl: 0.9-2.52) & 2.08 (95\% Cl: 1.77-2.39) \\
\hline Risk of ICU admission & 1.74 (95\% Cl: 1.35-2.09) & 2.23 (95\% Cl: 1.31-3.15) & 1.94 (95\% Cl: 1.71-2.18) & 3.35 (95\% Cl: 2.5-4.2) \\
\hline Risk of mortality & 1.37 (95\% Cl: 1.15-1.6) & 1.50 (95\% Cl: 1.26-1.74) & 1.06 (95\% Cl: 0.17-1.96) & 2.33 (95\% Cl: 1.45-3.21) \\
\hline
\end{tabular}

with the wild-type virus was 1.45 (95\% CI: $1.18-1.78)$, which was close to our meta-analysis result at 1.37 (95\% CI: $1.15-$ 1.60). A recent review concluded that variants Alpha, Beta and Gamma all had a higher risk of hospitalization and ICU admission compared with the wild-type virus, and the risk of Beta variant was much higher (23), which supported our conclusion to some extent.

To our knowledge, this is the first study to compare the disease severity of VOCs with the wild-type virus and draw specific conclusions. We believe that our results present the threats of VOCs more clearly to the public, particularly the variants Beta and Delta. Although several different types of vaccines have been developed, further research is required regarding the protection rate of the viral variants. The fact that we did not further analyze the influence of age, sex, and geographic parameters is the limitation of the study. However, we performed meta-analysis, which is known to better reduce the impact of each study.

\section{CONCLUSION}

In this meta-analysis, we analyzed the results of studies that reported on the disease severity of SARS-COV-2 VOCs from June 1, 2020 to October 15, 2021 and processed the relevant data. By comparing with the wild-type virus, in terms of the risk of hospitalization, ICU admission, and mortality, the variants Beta and Delta have a higher risk than the variants Alpha and Gamma, and all SARS-COV-2 VOCs have a higher risk of disease severity than the wild-type virus. This is the first comprehensive study that compared the disease severity of variants Alpha, Beta, Gamma and Delta with wildtype virus and drew specific conclusions. We hope that this report can increase the awareness of the disease severity of SARS-COV-2 VOCs, particularly of variants Beta and Delta, and make the public aware of routine precautions and the importance of vaccination.

\section{DATA AVAILABILITY STATEMENT}

The original contributions presented in the study are included in the article/supplementary material, further inquiries can be directed to the corresponding author/s.

\section{AUTHOR CONTRIBUTIONS}

All authors listed have made a substantial, direct, and intellectual contribution to the work and approved it for publication.

\section{FUNDING}

This work described in this paper was partially supported by a grant from the Research Grants Council of the Hong Kong Special Administrative Region, China (HKU C7123-20G). 


\section{REFERENCES}

1. Andrew R, Nick L, Oliver P, Wendy B, Jeff B, Alesandro C, et al. Preliminary genomic characterisation of an emergent SARS-CoV-2 lineage in the UK defined by a novel set of spike mutations. Genom. Epidemiol. (2020). Available online at: https://virological.org/t/preliminary-genomic-characterisationof-an-emergent-sars-cov-2-lineage-in-the-uk-defined-by-a-novel-set-ofspike-mutations/563 (accessed December 21, 2020)

2. Tegally H, Wilkinson E, Giovanetti M, Iranzadeh A, Fonseca V, Giandhari J, et al. Emergence and rapid spread of a new severe acute respiratory syndromerelated coronavirus 2 (SARS-CoV-2) lineage with multiple spike mutations in South Africa. medRxiv [Preprint]. (2020). doi: 10.1101/2020.12.21.20248640

3. Faria NR, Claro IM, Candido D, Franco LM, Andrade PS, Coletti TM, et al. Genomic characterisation of an emergent SARS-CoV-2 lineage in Manaus: preliminary findings. Virological. (2021) 372:815-21.

4. Adam D. What scientists know about new, fast-spreading coronavirus variants. Nature. (2021) 594:19-20. doi: 10.1038/d41586-021-01390-4

5. Buchan SA, Tibebu S, Daneman N, Whelan M, Vanniyasingam T, Murti $\mathrm{M}$, et al. Increased household secondary attacks rates with Variant of Concern SARS-CoV-2 index cases. medRxiv [Preprint]. (2021). doi: 10.1101/2021.03.31.21254502

6. Tegally H, Wilkinson E, Giovanetti M, Iranzadeh A, Fonseca V, Giandhari J, et al. Emergence of a SARS-CoV-2 variant of concern with mutations in spike glycoprotein. Nature. (2021) 592:438-43. doi: 10.1038/s41586-021-03402-9

7. Sinha S, Tam B, Wang SM. Altered interaction between RBD and ACE2 receptor contributes towards the increased transmissibility of SARS CoV-2 delta, kappa, beta, and gamma strains with RBD double mutations. bioRxiv [Preprint]. (2021). doi: 10.1101/2021.08.30.458303

8. Curran J, Dol J, Boulos L, Somerville M, McCulloch H, MacDonald $\mathrm{M}$, et al. Transmission characteristics of SARS-CoV-2 variants of concern Rapid Scoping Review. medRxiv [Preprint]. (2021). doi: $10.1101 / 2021.04 .23 .21255515$

9. Campbell F, Archer B, Laurenson-Schafer H, Jinnai Y, Konings F, Batra $\mathrm{N}$, et al. Increased transmissibility and global spread of SARS-CoV-2 variants of concern as at June 2021. Eurosurveillance. (2021) 26:2100509. doi: 10.2807/1560-7917.ES.2021.26.24.2100509

10. Dhar MS, Marwal R, Radhakrishnan V, Ponnusamy K, Jolly B, Bhoyar $\mathrm{RC}$, et al. Genomic characterization and epidemiology of an emerging SARS-CoV-2 variant in Delhi, India. medRxiv [Preprint]. (2021). doi: 10.1101/2021.06.02.21258076

11. Grabowski F, Preibisch G, Giziński S, Kochańczyk M, Lipniacki T. SARS-CoV-2 variant of concern 202012/01 has about twofold replicative advantage and acquires concerning mutations. Viruses. (2021) 13:392. doi: $10.3390 / \mathrm{v} 13030392$

12. Russell TW, Davies N, Kucharski AJ, group CC-w, Edmunds WJ, Eggo. RM, et al. Estimates of Severity and Transmissibility of Novel SARS-CoV-2 Variant 501Y.V2 in South Africa. CMMID (2021). Available online at: https://cmmid. github.io/topics/covid19/sa-novel-variant.html (accessed January 11, 2021)

13. Faria NR, Mellan TA, Whittaker C, Claro IM, Candido DdS, Mishra S, et al. Genomics and epidemiology of the P. 1 SARS-CoV-2 lineage in Manaus, Brazil. Science. (2021) 372:815-21. doi: 10.1126/science.abh2644

14. Emary KR, Golubchik T, Aley PK, Ariani CV, Angus B, Bibi S, et al. Efficacy of ChAdOx1 nCoV-19 (AZD1222) vaccine against SARSCoV-2 variant of concern 202012/01 $\quad$ (B. 1.1. 7): an exploratory analysis of a randomised controlled trial. Lancet. (2021) 397:1351-62. doi: 10.1016/S0140-6736(21)00628-0

15. Heath PT, Galiza EP, Baxter DN, Boffito M, Browne D, Burns F, et al. Efficacy of the NVX-CoV2373 Covid-19 vaccine against the B. 1.1. 7 variant. medRxiv [Preprint]. (2021). doi: 10.1101/2021.05.13.21256639

16. Madhi SA, Baillie V, Cutland CL, Voysey M, Koen AL, Fairlie L, et al. Efficacy of the ChAdOx1 nCoV-19 Covid-19 vaccine against the B. 1.351 variant. $N$ Engl J Med. (2021) 384:1885-98. doi: 10.1056/NEJMoa2102214

17. Sadoff J, Gray G, Vandebosch A, Cárdenas V, Shukarev G, Grinsztejn B, et al. Safety and efficacy of single-dose Ad26. COV2. S vaccine against Covid-19. N Engl J Med. (2021) 384:2187-201. doi: 10.1056/NEJMoa2101544

18. Shinde V, Bhikha S, Hossain Z, Archary M, Bhorat Q, Fairlie L, et al. Preliminary efficacy of the NVX-CoV2373 Covid-19 vaccine against the B. 1.351 variant. medRxiv [Preprint]. (2021). doi: 10.1101/2021.02.25.21252477
19. Thomas SJ, Moreira ED, Kitchin N, Absalon J, Gurtman A, Lockhart S, et al. Six Month safety and efficacy of the BNT162b2 Mrna Covid-19 vaccine. medRxiv [Preprint]. (2021). doi: 10.1101/2021.07.28.21261159

20. SARS-CoV-2 Variants of Concern and Variants under Investigation in England. Technical Briefing 10. Public Health England. Available online at: https:// assets.publishing.service.gov.uk/government/uploads/system/uploads/ attachment_data/file/984274/Variants_of_Concern_VOC_Technical_ Briefing_10_England.pdf (accessed May 7, 2021)

21. Ong SWX, Young BE, Lye DC. Lack of detail in population-level data impedes analysis of SARS-CoV-2 variants of concern and clinical outcomes. Lancet Infect Dis. (2021) 21:1195-7. doi: 10.1016/S1473-3099(21)00201-2

22. Kow CS, Merchant HA, Hasan SS. Mortality risk in patients infected with SARS-CoV-2 of the lineage B. 1.1. 7 in the UK. J Infect. (2021) 83:e14-5. doi: 10.1016/j.jinf.2021.05.008

23. Cantón R, De Lucas Ramos P, García-Botella A, García-Lledó A, GómezPavón J, González Del Castillo J, et al. New variants of SARS-CoV-2. Rev Esp Quimioter. (2021) 34:419-28. doi: 10.37201/req/071.2021

24. Moher D, Shamseer L, Clarke M, Ghersi D, Liberati A, Petticrew $\mathrm{M}$, et al. Preferred reporting items for systematic review and metaanalysis protocols (PRISMA-P) 2015 statement. Syst Rev. (2015) 4:1-9. doi: 10.1186/2046-4053-4-1

25. Frampton D, Rampling T, Cross A, Bailey H, Heaney J, Byott M, et al. Genomic characteristics and clinical effect of the emergent SARS-CoV-2 B. 1.1. 7 lineage in London, UK: a whole-genome sequencing and hospital-based cohort study. Lancet Infect Dis. (2021) 21:1246-56. doi: 10.1016/S1473-3099(21)00170-5

26. Challen R, Brooks-Pollock E, Read JM, Dyson L, Tsaneva-Atanasova $\mathrm{K}$, Danon L. Risk of mortality in patients infected with SARS-CoV-2 variant of concern 202012/1: matched cohort study. BMJ. (2021) 372:n579. doi: $10.1136 /$ bmj.n579

27. Davies NG, Jarvis CI, van Zandvoort K, Clifford S, Sun FY, Funk S, et al. Increased mortality in community-tested cases of SARS-CoV-2 lineage B.1.1.7. Nature. (2021) 593:270-4. doi: 10.1038/s41586-021-03426-1

28. Grint DJ, Wing K, Williamson E, McDonald HI, Bhaskaran K, Evans D, et al. Case fatality risk of the SARS-CoV-2 variant of concern B. 1.1. 7 in England, 16 November to 5 February. Eurosurveillance. (2021) 26:2100256. doi: 10.2807/1560-7917.ES.2021.26.11.2100256

29. Patone M, Thomas K, Hatch R, San Tan P, Coupland C, Liao W, et al. Analysis of severe outcomes associated with the SARS-CoV-2 Variant of Concern 202012/01 in England using ICNARC Case Mix Programme and QResearch databases. medRxiv [Preprint]. (2021). doi: 10.1101/2021.03.11.21253364

30. Loconsole D, Centrone F, Morcavallo C, Campanella S, Sallustio A, Accogli M, et al. Rapid spread of the SARS-CoV-2 Variant of Concern 202012/01 in Southern Italy (December 2020-March 2021). Int J Environ Res Public Health. (2021) 18:4766. doi: 10.3390/ijerph18094766

31. Funk T, Pharris A, Spiteri G, Bundle N, Melidou A, Carr M, et al. Characteristics of SARS-CoV-2 variants of concern B. 1.1. 7, B. 1.351 or P. 1: data from seven EU/EEA countries, weeks $38 / 2020$ to $10 / 2021$. Eurosurveillance. (2021) 26:2100348. doi: 10.2807/1560-7917.ES.2021.26.16.2100348

32. SARS-CoV-2 Variants of Concern and Variants under Investigation in England Technical Briefing 14. Public Health England (2021). Available online at: https://assets.publishing.service.gov.uk/government/uploads/ system/uploads/attachment_data/file/991343/Variants_of_Concern_VOC_ Technical_Briefing_14.pdf (accessed June 3, 2021)

33. Bager P, Wohlfahrt J, Fonager J, Rasmussen M, Albertsen M, Michaelsen TY, et al. Risk of hospitalisation associated with infection with SARS-CoV2 lineage B. 1.1. 7 in Denmark: an observational cohort study. Lancet Infect Dis. (2021) 21:1507-17. doi: 10.1016/S1473-3099(21)00290-5

34. Cetin M, Balci PO, Sivgin H, Cetin S, Ulgen A, Dörtok Demir H, et al. Alpha variant (B. 1.1. 7) of SARS-CoV-2 increases fatality-rate for patients under age of 70 years and hospitalization risk overall. Acta Microbiol Immunol Hungarica. (2021) 68:153-61. doi: 10.1556/030.2021. 01524

35. Fisman DN, Tuite AR. Evaluation of the relative virulence of novel SARSCoV-2 variants: a retrospective cohort study in Ontario, Canada. CMAJ. (2021) 193:E1619-E25. doi: 10.1503/cmaj.211248

36. Freitas ARR, Beckedorff OA, Cavalcanti LPdG, Siqueira AM, Castro $\mathrm{DBd}$, Costa $\mathrm{CFd}$, et al. The emergence of novel SARS-CoV-2 variant 
P.1 in Amazonas (Brazil) was temporally associated with a change in the age and sex profile of COVID-19 mortality: a population based ecological study. The Lancet Regional Health - Americas. (2021) 1:100021. doi: 10.1016/j.lana.2021.100021

37. Grint DJ, Wing K, Houlihan C, Gibbs HP, Evans SJ, Williamson E, et al. Severity of SARS-CoV-2 alpha variant (B. 1.1. 7) in England. Clin Infect Dis. (2021) ciab754. doi: 10.1093/cid/ciab754

38. Giles B, Meredith P, Robson S, Smith G, Chauhan A. The SARS-CoV-2 B. 1.1. 7 variant and increased clinical severity-the jury is out. Lancet Infect Dis. (2021) 21:1213-4. doi: 10.1016/S1473-3099(21)00356-X

39. Hoang V-T, Colson P, Levasseur A, Delerce J, Lagier J-C, Parola P, et al. Clinical outcomes in patients infected with different SARS-CoV-2 variants at one hospital during three phases of the COVID-19 epidemic in Marseille, France. Infect Genet Evol. (2021) 95:105092. doi: 10.1016/j.meegid.2021.105092

40. Kim Y, Kim E-J, Lee S-W, Kwon D. Review of the early reports of the epidemiological characteristics of the B. 1.1. 7 variant of SARS-CoV-2 and its spread worldwide. Osong Public Health Res Perspect. (2021) 12:139-48. doi: $10.24171 /$ j.phrp.2021.0037

41. Meyer M, Holfter A, Ruebsteck E, Gruell H, Dewald F, Koerner RW, et al. The Alpha Variant (B. 1.1. 7) of SARS-CoV-2 in children: first experience from 3544 nucleic acid amplification tests in a cohort of children in Germany. Viruses. (2021) 13:1600. doi: 10.3390/v13081600

42. Ong SWX, Chiew CJ, Ang LW, Mak T-M, Cui L, Toh MPH, et al. Clinical and virological features of SARS-CoV-2 variants of concern: a retrospective cohort study comparing B. 1.1. 7 (Alpha), B. 1.315 (Beta), and B. 1.617. 2 (Delta). Clin Infect Dis. (2021) ciab721. doi: 10.2139/ssrn.3861566

43. Martínez-García L, Espinel MA, Abreu M, González-Alba JM, Gijón D, McGee A, et al. Emergence and spread of B. 1.1. 7 lineage in primary care and clinical impact in the morbi-mortality among hospitalized patients in Madrid, Spain. Microorganisms. (2021) 9:1517. doi: 10.3390/microorganisms9071517

44. Yilmaz H, Cakir M, Komurcu SZM, Kazezoglu C, Guner AE, Memisoglu $\mathrm{K}$, et al. Evaluation of patients with COVID-19 and the United Kingdom mutations in a training and research hospital in Istanbul. Northern Clin Istanbul. (2021) 8:317. doi: 10.14744/nci.2021.90947

45. Twohig KA, Nyberg T, Zaidi A, Thelwall S, Sinnathamby MA, Aliabadi S, et al. Hospital admission and emergency care attendance risk for SARS-CoV-2 delta (B. 1.617. 2) compared with alpha (B. 1.1. 7) variants of concern: a cohort study. Lancet Infect Dis. (2021). doi: 10.1016/S1473-3099(21)00475-8

46. Veneti L, Seppälä E, Larsdatter Storm M, Valcarcel Salamanca B, Alnes Buanes E, Aasand N, et al. Increased risk of hospitalisation and intensive care admission associated with reported cases of SARS-CoV-2 variants B.
1.1. 7 and B. 1.351 in Norway, December 2020-May 2021. PLoS One. (2021) 16:e0258513. doi: 10.1371/journal.pone.0258513

47. Patone M, Thomas K, Hatch R, San Tan P, Coupland C, Liao W, et al. Mortality and critical care unit admission associated with the SARS-CoV-2 lineage B. 1.1. 7 in England: an observational cohort study. Lancet Infect Dis. (2021) 21:1518-28. doi: 10.1016/S1473-3099(21)00318-2

48. Nyberg T, Twohig KA, Harris RJ, Seaman SR, Flannagan J, Allen H, et al. Increased risk of hospitalisation for COVID-19 patients infected with SARS-CoV-2 variant B. 1.1. 7. [arXiv preprint] arXiv:05560. (2021). doi: 10.1136/bmj.n1412

49. Stirrup O, Boshier F, Venturini C, Guerra-Assunção JA, Alcolea-Medina A, Beckett A, et al. SARS-CoV-2 lineage B. 1.1. 7 is associated with greater disease severity among hospitalised women but not men: multicentre cohort study. BMJ Open Respir Res. (2021) 8:e001029. doi: 10.1136/bmjresp-2021-001029

50. Whittaker R, Kristofferson AB, Seppälä E, Salamanca BV, Veneti L, Storm ML, et al. Trajectories of hospitalisation for patients infected with SARS-CoV2 variant B. 1.1. 7 in Norway, December 2020-April 2021. J Infect. (2021) 83:e14-e7. doi: 10.1016/j.jinf.2021.07.025

51. Lei Y, Zhang J, Schiavon CR, He M, Chen L, Shen H, et al. SARS-CoV-2 spike protein impairs endothelial function via downregulation of ACE 2. Circ Res. (2021) 128:1323-6. doi: 10.1161/CIRCRESAHA.121.318902

52. Zhang X, Li S, Niu S. ACE2 and COVID-19 and the resulting ARDS. Postgrad Med J. (2020) 96:403-7. doi: 10.1136/postgradmedj-2020-137935

Conflict of Interest: The authors declare that the research was conducted in the absence of any commercial or financial relationships that could be construed as a potential conflict of interest.

Publisher's Note: All claims expressed in this article are solely those of the authors and do not necessarily represent those of their affiliated organizations, or those of the publisher, the editors and the reviewers. Any product that may be evaluated in this article, or claim that may be made by its manufacturer, is not guaranteed or endorsed by the publisher.

Copyright (c) 2021 Lin, Liu, Tang and He. This is an open-access article distributed under the terms of the Creative Commons Attribution License (CC BY). The use, distribution or reproduction in other forums is permitted, provided the original author(s) and the copyright owner(s) are credited and that the original publication in this journal is cited, in accordance with accepted academic practice. No use, distribution or reproduction is permitted which does not comply with these terms. 